\title{
Estrategias discursivas en la publicidad audiovisual de productos de bajo valor nutricional dirigidos al público infantil: felices, valientes y obesos*
}

\author{
Mónika Jiménez-Morales ${ }^{1}$ \\ Mireia Montaña ${ }^{2}$ \\ Mercè Vàzquez ${ }^{3}$
}

Recibido: 04/05/2018

Aprobado por pares: 11/07/2018
Enviado a pares: 22/05/2018

Aceptado: 11/10/2018

DOI: 10.5294/pacla.2019.22.3.10

Para citar este artículo / to reference this article / para citar este artigo Jiménez-Morales, M., Montaña, M. y Vàzquez, M. (2019). Estrategias discursivas en la publicidad audiovisual de productos de bajo valor nutricional dirigidos al público infantil: felices, valientes y obesos. Palabra Clave, 22(3), e22310. D0I: http://doi.org/10.5294/ pacla.2019.22.3.10

\section{Resumen}

Los niños españoles ven una media de 9000 campañas publicitarias en televisión al año. La mayoría de ellas corresponden a alimentos de bajo valor nutricional. Dados los índices de obesidad infantil, la estrecha relación entre la publicidad dirigida a ese colectivo y el consumo de este tipo de alimentos, España aprobó en 2005 el Código de Autorregulación de la Publicidad de Alimentos Dirigida a Menores (PAOS). Este artículo tiene como objetivo analizar las estrategias discursivas utilizadas en las campañas de alimentos de bajo valor nutricional, poniéndolas en relación con el contenido

\footnotetext{
* Los datos son fruto del trabajo vinculado al proyecto I+D+I del Ministerio de Economía y Competitividad de España (Mineco) CSO 2014-58220-r: "representación mediática de la imagen corporal no saludable. Desarrollo de una herramienta de prevención en niños y niñas de 5 a 8 años: ¡mi cuerpo me gusta!” (Mediacorp).

1 orcid.org/0000-0002-4977-0722. Universitat Pompeu Fabra, España. monika.jimenez@upf.edu

2 orcid.org/0000-0002-6464-5129. Universitat Oberta de Catalunya, España. mmontanabl@uoc.edu

3 orcid.org/0000-0002-7983-4029. Universitat Oberta de Catalunya, España. mvazquezga@uoc.edu
} 
del Código PAOS. Utiliza para ello una metodología mixta que integra técnicas cuantitativas para el análisis de audiencias y cualitativas para el estudio del discurso publicitario. La investigación concluye que, a diferencia de otro tipo de alimentos, la publicidad de productos de bajo valor nutricional centra su estrategia discursiva en palabras y campos semánticos vinculados a la felicidad, la acción y la diversión, entre otros. Las conclusiones apuntan asimismo a la necesidad de revisar el contenido del Código PAOS, de una mayor implicación por parte de la industria alimentaria española, de agencias y de anunciantes, con el objetivo de que la autorregulación de los contenidos publicitarios resulte realmente efectiva. La contribución original del artículo radica en la aportación teórica y empírica que toma como punto de partida la obesidad infantil en España y la interrelaciona con las estrategias discursivas de la publicidad de productos de bajo valor nutricional dirigida a los menores y el marco normativo existente.

\section{Palabras clave (fuente: tesauro de la Unesco)}

Publicidad; alimento; producto alimenticio; industria alimentaria; nutrición; niño; obesidad; obesidad infantil; lenguaje; discurso; emociones. 


\section{Discursive Strategies in Child-Directed Audiovisual Advertising of Low Nutritional Value Products: Happiness, Courage and Obesity*}

\section{Abstract}

Spanish children see an average of 9,000 advertising campaigns on television per year, mostly for low nutritional value foods. Given childhood obesity rates, the close relationship between advertising targeting this group and consumption of this type of food, Spain passed the Child-Directed Food Advertising Self-Regulation (PAOS) Code in 2005. This article intends to analyze the discursive strategies used in low nutritional value food campaigns in relation to the contents of the PAOS Code. It uses a mixed method that integrates quantitative techniques to analyze audiences and qualitative ones to study the advertising discourse. Research concludes that, unlike other types of foods, low nutritional value product advertising focuses its discursive strategy on words and semantic fields linked to happiness, action, fun, etc. Conclusions also point to the need to review the contents of the PAOS Code and further involve the Spanish food industry, agencies, and advertisers so that advertising content self-regulation becomes truly effective. The original contribution of this article lies in the theoretical and empirical inputs that take childhood obesity in Spain as a starting point and interrelate it with discursive strategies employed in low nutritional value product advertising aimed at minors and the existing regulatory framework.

\section{Keywords (Source: Unesco Thesaurus)}

Advertising; food; food products; food industry; nutrition; children; obesity; childhood overweight; language; discourse; emotions.

\footnotetext{
* The data collected was thanks to the I+D+I project of the Ministerio de Economía y Competitividad (Mineco) of Spain: CSO 2014-58220-r: mediatic representation of the image of a non-healthy body. Development of a prevention tool in kids of 5 to 8 years: "Mi cuerpo me gusta" (Mediacorp).
} 


\section{Estratégias discursivas na publicidade audiovisual de produtos de baixo valor nutricional dirigidos ao público infantil: felizes, corajosos e obesos*}

\section{Resumo}

As crianças espanholas vêm, em média, nove mil campanhas publicitárias pela televisão por ano. A maioria delas corresponde a alimentos de baixo valor nutricional. Tendo em vista os índices de obesidade infantil, a estreita relação entre a publicidade dirigida a esse público e o consumo desse tipo de alimento, a Espanha aprovou, em 2005, o Código de Autorregulamentação da Publicidade de Alimentos dirigida a Menores (PAOS). Este artigo tem como objetivo analisar as estratégias discursivas utilizadas nas campanhas de alimentos de baixo valor nutricional, colocando-as em relação com o conteúdo do Código PAOS. Para isso, utiliza uma metodologia mista, que integra técnicas quantitativas para a análise de audiências e qualitativas para o estudo do discurso publicitário. Esta pesquisa conclui que, à diferença de outros alimentos, a publicidade de produtos de baixo valor nutricional centraliza sua estratégia discursiva em palavras e campos semânticos vinculados à felicidade, à ação e à diversão, entre outros. As conclusões apontam, ainda, a necessidade de revisão do conteúdo do Código PAOS por parte da indústria alimentícia espanhola, de agências e de anunciantes, a fim de que a autorregulamentação dos conteúdos publicitários seja realmente efetiva. A contribuição original deste artigo está no campo teórico e empírico que toma como ponto de partida a obesidade infantil na Espanha e a inter-relaciona com as estratégias discursivas da publicidade de produtos de baixo valor nutricional dirigida aos menores e com o referencial normativo existente.

* Os dados são o resultado do trabalho ligado ao projeto I+D+I do Ministerio de Economía y Competitividad (Mineco) da Espanha: CSO 2014-58220-r: Representação midiática da imagem corporal não saudável. Desenvolvimento de uma ferramenta de prevenção para crianças de 5 a 8 anos de idade: “¡mi cuerpo me gusta!” (Mediacorp). 


\section{Palavras-chave (Fonte: tesauro da Unesco)}

Publicidade; alimento; produto alimentício; indústria de alimentos; nutrição; criança; obesidade; obesidade infantil; linguagem; discurso; emoções. 


\section{Introducción}

La prevalencia de la obesidad infantil en España, donde aproximadamente uno de cada tres niños tiene sobrepeso, se encuentra entre las más altas de Europa. Esta patología infantil es uno de los mayores problemas con los que se enfrenta la salud pública actual de los países desarrollados, y puede repercutir, aparte de en exceso de grasa y sus consecuencias psicológicas, en otras enfermedades relacionadas en la edad infantil o adulta (Baker, Olsen \& Sørensen, 2007; Bibbins-Domingo, Coxson, Pletcher, Lightwood \& Goldman, 2007; Ensenyat, Palacios, Serra-Paya \& Castro-Viñuales, 2016).

Aunque hay pocas investigaciones que asocien directamente la publicidad de alimentos con la obesidad infantil, hay una fuerte evidencia de la relación entre consumir-televisión y una mayor ingesta de alimentos de alta densidad energética y la obesidad (Lee, Kim, Lee, Yoon \& Chung, 2014); de hecho, la televisión sigue siendo el principal canal para la comercialización de alimentos y bebidas para los niños, en especial en las edades más tempranas (Royo-Bordonada et al., 2016). Cabe señalar que la mayoría de los niños menores de 6 años aún no saben distinguir entre la programación y la publicidad, y que los niños menores de 8 años tampoco entienden la intención persuasiva de los mensajes publicitarios (Campos et al., 2016).

Dentro de la totalidad de anuncios que ven los niños al año, predominan los de alimentos catalogados como poco saludables dado su alto contenido en azúcares, grasas o sal (González, 2013a, 2013b; Royo-Bordonada et al., 2016), de manera que hay una mayor exposición televisiva a la comida considerada poco saludable que a la alimentación sana.

Con el objetivo de reducir las alarmantes tasas de obesidad infantil en España, en 2005 entró en vigor la estrategia NAOS (nutrición, actividad física y prevención de la obesidad) promovida desde el Ministerio de Sanidad y Consumo, a través de la Agencia Española de Seguridad Alimentaria y Nutrición (Aesan). Esta acción tiene como objetivo sensibilizar a la población del problema que la obesidad representa para la salud e impulsar iniciativas que contribuyan a lograr que los ciudadanos, y en especial los niños y los jóvenes, adopten hábitos de vida saludables. En el marco de dicha es- 
trategia, se insertó el Código de autorregulación de la publicidad de alimentos y bebidas dirigida a menores, prevención de la obesidad y salud (PAOS), normativa de autorregulación que establece las reglas que rigen el desarrollo, la ejecución y la difusión de los mensajes publicitarios dirigidos a menores de 12 años. Su objetivo es el de proteger a los niños a partir de ciertas limitaciones en la comunicación de los productos destinados a ellos.

El Código PAOS supuso en su momento un gran avance en la regulación de la publicidad infantil de alimentos y fijaba los mecanismos que garantizaban el control y la aplicación de estas normas. Dentro de sus normas éticas, aparecía, entre otras, la limitación de que la publicidad de alimentos o bebidas en ningún caso explotaría la confianza de los menores en sus padres, en sus profesores o en otras personas (reales o ficticias) de películas o series de ficción. Con ello, se pretendía evitar que la presencia o el testimonio de determinadas personas o personajes admirados por los menores en anuncios dirigidos a ellos ejerciera una influencia indebida sobre los menores. De esta manera, se buscaba que los menores no se pudieran ver impulsados a solicitar la compra de un producto alimenticio anunciado, no tanto por las características del producto, sino por el simple hecho del testimonio o soporte del personaje del anuncio. Cabe señalar que en 2012 el Código PAOS fue actualizado, reconsiderando aquellos aspectos que originariamente no se tuvieron en cuenta (Martín, Fernández-Poyatos y OrtizMoncada, 2011). Actualmente, estas normas de autorregulación amplían hasta los 15 años la edad de protección del público, y consideran la publicidad en internet, teniendo en cuenta el marketing viral, entre otros, o la protección de datos (González, 2013a).

Aun así, en la actualidad, el código presenta ciertas carencias, como el hecho de que, a pesar de instar al anunciante a mostrar el producto junto con una variedad razonable de alimentos para fomentar una dieta equilibrada (González, 2013b), no regule la calidad nutricional de los productos anunciados, ni tenga en cuenta los potenciales efectos negativos del consumo de esos productos (Ponce-Blandón, Pabón-Carrasco y Lomas-Campos, 2017) o la frecuencia de emisión de este tipo de anuncios (Royo-Bordonada et al., 2016). Tampoco tiene en cuenta el discurso 
utilizado y las emociones positivas que pueden asociarse al lenguaje usado en los anuncios de alimentación como posible factor de influencia en la decisión de compra.

Aunque existe una legislación específica, se evidencia que la normativa española de control de la publicidad de alimentos, y la de la mayor parte de países del mundo, continúa siendo insuficientes para evitar la publicidad de alimentos poco saludables en televisión y, en consecuencia, para facilitar la prevención temprana de la obesidad (Campos et al., 2016; González, 2013a; Crovetto, Durán, Guzmán y Miranda, 2011). Durante el visionado de productos alimenticios en televisión, se activan áreas específicas del cerebro, en especial cuando el mensaje emitido se asocia el placer y las experiencias gratificantes (Campos et al., 2016). Este hecho resulta determinante en el proceso de decisión de compra y en el posterior consumo.

De hecho, algunas teorías apuntan a una relación positiva existente entre la información que proporcionan los anuncios y el placer encontrado (Hyun, Kim \& Lee, 2011). Estos estudios afirman que otro de los factores que provocan respuestas positivas al mensaje es la estimulación. Por otra parte, la empatía también obtiene resultados altamente positivos: cuando un consumidor empatiza con una persona, objeto o actividad representada en el anuncio, siente una implicación emocional que le ayuda a crear creencias positivas sobre el producto o servicio anunciado. La familiaridad, obtenida a menudo por el uso de famosos en los anuncios, también provoca respuestas emocionales positivas aumentando la efectividad de los anuncios.

Diferentes estudios interrelacionan el consumo de alimentos de bajo valor nutricional con los bajos estados de ánimo. Asimismo, estas investigaciones utilizan el concepto de alimentación emocional para designar la ingesta de alimentos con el objetivo de suplir carencias emocionales. Tal como constatan Oliver, Wardle \& Gibson (2016), los llamados comedores emocionales consumen más alimentos ricos en energía en respuesta a las emociones negativas que el resto de la población. De esa manera, no resulta baladí el hecho de que la publicidad de alimentos de bajo 
valor nutricional tienda a basar su estrategia persuasiva en la promesa de experiencias eminentemente positivas que se transmiten a partir del lenguaje, tal como pretende poner de manifiesto la investigación que presentamos a continuación.

En relación con este hecho, cabe señalar que estudios anteriores realizados en España ya habían observado una forma de reclamo en especial perjudicial para los niños: asociar la ingesta de un determinado alimento en ser mejores (Menéndez y Franco, 2009). Igualmente, algunos elementos persuasivos de tipo emocional han demostrado tener un papel muy relevante en la configuración de los mensajes publicitarios (Ponce-Blandón, Pabón-Carrasco y Lomas-Campos, 2017).

Son diversos los estudios que ponen de manifiesto la vulnerabilidad del colectivo infantil ante ciertos estímulos presentes en la publicidad de alimentos dirigidos en especial a ese público (González, 2013a; Kelly et al., 2010; Sevillano y Sotomayor, 2012). Estas investigaciones constatan la relación entre los anuncios de alimentos que ven los niños y el consumo de los productos anunciados.

En relación a esta cuestión, aspectos como el placer, la felicidad y, en definitiva, todos aquellos elementos hedónicos presentes en el discurso publicitario de ciertos productos revierten positivamente en el consumo, ya que, a través de la compra de ese producto, el público busca inconscientemente mantener ese estado de felicidad que promete la narrativa publicitaria (Eunjin et al., 2017). En cualquier caso, se trata de estrategias ajenas al producto en sí que en muchas ocasiones pretenden maquillar; en el caso de los productos alimenticios, sus carencias nutricionales (Ponce-Blandón, Pabón-Carrasco y Lomas-Campos, 2017).

Otras investigaciones afirman que tanto la comunicación alimentaria basada en el hedonismo como el embalaje de los productos desempeñan un papel fundamental en el desorden alimentario de vinculación de comida con emociones. En la mayoría de casos, esta vinculación a la comida emocional se desarrolla durante la infancia (Kemp, Bui \& Grier, 2013). 
Los anuncios generan imágenes mentales que inciden en el recuerdo y en la evocación de respuestas por parte de los públicos y, en ese último aspecto, los estímulos verbales no abstractos y de clase abierta (nombres, verbos y adjetivos) favorecen un mayor recuerdo y una actitud favorable hacia la marca, despertando la intención de compra (Arroyo-Almaraz y García-García, 2015).

Si bien son diversos los estudios que analizan el lenguaje de la publicidad de alimentos (Fuertes-Olivera et al., 2001; Kim, Ratneshwar \& Thorson, 2017; McGuire, 2000; Prell et al., 2011; Teodorescu, 20015), resultan muy pocos aquellos que recaban en el léxico utilizado en los anuncios de productos de bajo valor nutricional (Jenkin, Madhvani, Signal \& Bowers, 2014) y ninguno centrado en la cuestión de si este lenguaje presenta diferencias respecto de las campañas publicitarias de otro tipo de alimentos.

El presente artículo recoge la investigación llevada a cabo para determinar la interrelación existente entre los anuncios más vistos por los niños españoles durante 2015, los estándares nutricionales de los productos alimentarios publicitados, el lenguaje utilizado en dicha publicidad a modo de reclamo y la relación de todos esos elementos con la normativa de autorregulación que se recoge en el Código PAOS.

\section{Metodología}

Con el propósito de identificar los diferentes elementos que definen los objetivos de esta investigación, hemos utilizado una metodología mixta, que integra técnicas cuantitativas y cualitativas. En ese sentido, hemos trabajado de forma cuantitativa con los datos de audiencia de Kantar Media. Hemos determinado la muestra a partir de la segmentación por franjas horarias y por cadenas para determinar el consumo televisivo de los niños españoles de entre 4 a 12 años durante 2015. Cabe señalar que Kantar Media no establece franjas de edad en lo que denomina la categoría infantil; por ese motivo, la edad de la muestra seleccionada se ciñe a la categorización de la fuente de datos, es decir, a la comprendida entre los 4 y los 12 años. Por otra parte, a diferencia de estudios previos centrados únicamente en cadenas infantiles, en la presente investigación analizamos la 
publicidad y la audiencia de todos los canales de televisión convencional que operan en España.

Las preguntas de investigación que han determinado el estudio son, en primer lugar, cuáles han sido los anuncios de alimentación más vistos por los niños de entre 4 y 12 años durante 2015; en segundo lugar, cómo es el discurso utilizado en estas campañas; y en tercer lugar, hasta qué punto las estrategias discursivas utilizadas en la publicidad de alimentos se ajustan al Código PAOS.

Con el objetivo de responder a la primera pregunta de investigación, se ha elaborado un ranking de la publicidad televisiva de productos de alimentación más visionada por los niños de esa edad. Una vez hemos obtenido el listado de los anuncios de esa categoría más vistos por este colectivo hemos buscado las piezas publicitarias en Infoadex Mosaico, con el fin de efectuar el análisis de contenido que corresponde a la aplicación de la metodología cualitativa.

Posteriormente, para responder a la segunda pregunta de investigación y determinar las características del discurso empleado, hemos transcrito el mensaje publicitario de cada una de las campañas, un total de 100. A partir de la transcripción realizada, hemos diseñado una ficha de análisis de las unidades léxicas más utilizadas en los anuncios dirigidos al público de referencia. Para alcanzar los objetivos de la investigación, en el análisis de dichas unidades léxicas se han tenido en cuenta las siguientes variables: producto, categoría de alimento, valor nutricional, campo semántico, contexto en el que se utiliza el discurso y creación léxica.

Las dos primeras variables - producto y categoría de alimentonos han servido para identificar (nombre) y clasificar el producto en función de su tipología (lácteo, bollería, etc.). También hemos introducido el valor nutricional de los productos como variable de análisis, utilizando para ello la Base de Datos Española de Composición de Alimentos (Bedca). Esta variable nos ha permitido diferenciar los productos naturales de aquellos con azúcar añadido y un alto contenido en carbohidratos (Ortiz- 
Pérez et al., 2015), y clasificarlos posteriormente en productos de alto, medio o bajo valor nutricional.

Hemos introducido la variable de campos semánticos para poder aglutinar las palabras que aparecen en el discurso y que se refieren a un mismo tema, con el objetivo de determinar si estas unidades léxicas corresponden realmente al campo semántico de la alimentación, o si más bien se incluyen dentro de aquellos que atañen a estados emocionales o a experiencias positivas.

La variable de contexto tiene como objetivo saber en qué circunstancias y condiciones se enmarca el discurso publicitario analizado (el producto anunciado se consume en la mesa, durante una actividad deportiva, en un momento de diversión entre amigos, etc.). Por su parte, la variable de creación léxica pretende determinar si se crean palabras a partir del nombre de la marca o el producto, y las acciones o situaciones mostradas en el anuncio. A partir de esta variable, pretendemos determinar si los neologismos que aparecen en la publicidad de alimentos de bajo valor nutricional están relacionados con el propio producto o si, por el contrario, están vinculados a estados de ánimo u otros elementos alejados de este (De Iulio, 2010).

En paralelo, con el propósito de responder a la tercera pregunta de investigación, se ha procedido al análisis del contenido de los artículos que integran el Código PAOS, a fin de determinar si las estrategias discursivas utilizadas por la publicidad de alimentos de bajo valor nutricional se ajus$\tan$ a este. En ese sentido, se toman en consideración los siguientes criterios del Código PAOS: el tipo de producto alimenticio promocionado y el diseño del mensaje publicitario. Asimismo, se analiza la concordancia del discurso publicitario con las diferentes normas éticas: principio de lealtad, educación e información nutricional, presentación de los productos, información sobre los productos, presión de las ventas, apoyo y promoción a través de personajes y programas, identificación de la publicidad, presentaciones comparativas, promociones, sorteos y clubes infantiles, seguridad, tratamiento de datos personales, marketing viral y protección frente a contenidos inapropiados. 


\section{Resultados}

Los datos obtenidos a partir de la segmentación por audiencias, franjas horarias y por cadenas de Kantar Media y de la Asociación de Usuarios de la Comunicación, el consumo de televisión por parte de los menores se situó en una media de 133 min diarios en 2015. La franja televisiva de mayor audiencia, de lunes a viernes, entre los menores se sitúa en prime time entre las 22:00 y las 24:00 h, alcanzando un 18,3\% de este público objetivo. Este porcentaje supone más de 1200000 menores expuestos al medio televisivo a partir de las 10 p. m., fuera del horario de protección, es decir, de 8 a $9 \mathrm{~h}$ y de 17 a 20 h entre semana, y de 9 a 12 h el fin de semana.

La segunda franja de mayor audiencia se sitúa entre las 20:00 h y las 22:00 h con un 16,9\%. Le sigue la franja entre las 13:00 h y las 17:00 h $(12,9 \%)$. Es destacable que las franjas de protección infantil coinciden en momentos de menor audiencia, tanto por la mañana $(5,4 \%)$ como por la noche $(12,0 \%)$. También se debe tener muy en cuenta la forma de consumo televisivo de los menores; según estos datos, hasta un 32,2\% de los niños consumen televisión sin la compañía y supervisión de un adulto. Según Kantar Media, los niños de 4 a 12 años españoles recibieron una presión publicitaria televisiva total de 1444772 gross ratings point (GRP) o número de exposiciones durante 2015. La categoría de producto que más impactó este público objetivo ha sido la de productos de alimentación, con un $18 \%$ de los GRP totales, seguido de la de deporte y tiempo libre (14\% GRP) y belleza e higiene (11\% GRP).

Teniendo en cuenta estos resultados, hemos analizado los anuncios más vistos por los niños de 4 a 12 años correspondientes a la categoría alimentación, que es la que tuvo más impacto en este segmento de población. Para realizar este análisis, hemos clasificado los distintos productos anunciados teniendo en cuenta las siguientes variables: alimento, categoría de alimento, valor nutricional, unidades léxicas utilizadas en el anuncio, campo semántico de dichas unidades y creación léxica.

En cuanto a los alimentos, de un total de 100 campañas analizadas, hemos identificado 54 tipos de alimentos, que han sido clasificados en 17 categorías de alimentos (tabla 1). 


\section{Tabla 1. Categorías de alimentos}

\begin{tabular}{|c|c|}
\hline Categorías de alimentos & Productos \\
\hline Arroz & Arroz SOS \\
\hline Bollería & Phoskitos, La Bella Easo Weikis, Bollycao, Bollycao Wap’s, Bimbo Kekos \\
\hline Cacao instantáneo & Nesquik, Colacao \\
\hline Caldo & Caldo casero Gallina Blanca \\
\hline Caramelos & Tic tac \\
\hline Cereales & Chocapic, Choco Flakes, Choco Krispis, Lion \\
\hline Chocolate & Nocilla, Lacasitos, Lacasitos White, Nutella, Paladín Minuto \\
\hline Fruta & Plátano de Canarias \\
\hline Galletas & $\begin{array}{l}\text { Tosta Rica, Dinosaurus, Chiquilin, Tosta Rica Oceanix, Dinosaurus huevo, Princesa, Príncipe } \\
\text { Galletas, Oreo, Mini galletas Arluy }\end{array}$ \\
\hline Helado & Pirulo Jungly, Maxibón, Frigo Max Calippo, Max Music Tornado \\
\hline Lácteos & $\begin{array}{l}\text { Actimel, Activia, Danonino, Danone sabores, Danet, Danet Doble Placer, Batido Leche Pascual } \\
\text { Yogur para beber de Danone }\end{array}$ \\
\hline Leche & Puleva Max, Almirón Advance \\
\hline Pasta & Yatekomo, Pastas Gallo \\
\hline Patatas & Pringles, Cheetos Moovins \\
\hline Paté & La Piara \\
\hline Queso & Mini Babybel, La vaca que ríe, La vaca que ríe campesino, Kiri, Crema de queso President \\
\hline Salchichas & Ketchup Plop's \\
\hline
\end{tabular}

Fuente: elaboración propia.

Asimismo, hemos tenido en cuenta el valor nutricional de cada alimento aplicando los criterios de la Bedca, diferenciando los productos naturales de aquellos con azúcar añadido y un alto contenido en carbohidratos (Ortiz-Pérez et al., 2015) y clasificándolos en productos de alto, medio o bajo valor nutricional. En nuestro caso, el valor nutricional del conjunto de alimentos analizados se sitúa entre los valores medio y bajo, puesto que solo un alimento tiene asociado un valor nutricional alto. Así pues, de los 54 tipos de alimentos identificados, 1 tiene un valor nutricional alto, 17 de ellos tienen un valor nutricional medio y los 36 restantes tienen un valor nutricional bajo. El valor nutricional alto corresponde a un alimento natural, fruta en este caso, mientras que los valores medios están relacionados con los productos lácteos y los quesos, y los de bajo valor coinciden con las galletas, los cereales, la bollería o el cacao instantáneo, entre otros. 
Además, hemos definido un conjunto de campos semánticos que son representativos de las unidades léxicas utilizadas en los anuncios publicitarios y que muestran un núcleo común de rasgos de significado entre dichas unidades. La creación de estos campos semánticos nos ha permitido identificar los rasgos más característicos de los alimentos a partir de las distintas unidades léxicas que tienen asociadas, y también establecer una correlación entre la categoría del alimento, el valor nutricional y el campo semántico en el que se sitúan las unidades léxicas que describen los alimentos de cada categoría. Hemos observado que los campos semánticos más utilizados en el conjunto de anuncios analizados hacen referencia a las cualidades de los alimentos en su gran mayoría ( 66 anuncios), a ocio (54 anuncios), a estados de ánimo (32 anuncios), a comida ( 16 anuncios), a chocolate y dulces ( 15 anuncios) o a lácteos, objetos, panadería y pastelería (10 anuncios de cada uno).

A continuación, mostramos las 17 categorías de alimentos a las que pertenecen los productos de alimentación que aparecen en los anuncios de la muestra analizada, junto con su valor nutricional, el campo semántico al que pertenecen las unidades léxicas utilizadas en los anuncios y las correspondientes unidades léxicas clasificadas por categoría gramatical (tabla 2).

\section{Tabla 2. Categoría de alimentos, valor nutricional, campos semánticos y unidades léxicas}

\begin{tabular}{|c|c|c|c|c|c|}
\hline $\begin{array}{c}\text { Categorías } \\
\text { de alimentos }\end{array}$ & $\begin{array}{c}\text { Valor } \\
\text { nutricional }\end{array}$ & Campos semánticos & $\begin{array}{c}\text { Unidad léxica: } \\
\text { sustantivo }\end{array}$ & $\begin{array}{c}\text { Unidad léxica: } \\
\text { verbo }\end{array}$ & $\begin{array}{c}\text { Unidad léxica: } \\
\text { adjetivo }\end{array}$ \\
\hline \multirow{3}{*}{ Arroz } & \multirow{3}{*}{ Medio } & Cualidades de los alimentos & & & Bueno \\
\hline & & Cereales & Arroz & & \\
\hline & & Estados de ánimo & & Disfrutar & \\
\hline \multirow{7}{*}{ Bollería } & \multirow{7}{*}{ Bajo } & Cualidades de los alimentos & Hierro & & $\begin{array}{c}\text { Delicioso } \\
\text { Bueno } \\
\text { Sano }\end{array}$ \\
\hline & & Cereales & Cereales & & \\
\hline & & Chocolate y dulces & $\begin{array}{l}\text { Chocolate } \\
\text { Bizcochito } \\
\text { Bizcocho }\end{array}$ & & \\
\hline & & Comida & $\begin{array}{l}\text { Desayuno } \\
\text { Merienda }\end{array}$ & & \\
\hline & & Ingerir & & Comer & \\
\hline & & Estados de ánimo & Vitalidad & Disfrutar & \\
\hline & & Ocio & Concurso & & \\
\hline
\end{tabular}




\begin{tabular}{|c|c|c|c|c|c|}
\hline $\begin{array}{c}\text { Categorías } \\
\text { de alimentos }\end{array}$ & $\begin{array}{c}\text { Valor } \\
\text { nutricional }\end{array}$ & Campos semánticos & $\begin{array}{c}\text { Unidad léxica: } \\
\text { sustantivo }\end{array}$ & $\begin{array}{c}\text { Unidad léxica: } \\
\text { verbo }\end{array}$ & $\begin{array}{c}\text { Unidad léxica: } \\
\text { adjetivo }\end{array}$ \\
\hline \multirow{10}{*}{$\begin{array}{c}\text { Cacao } \\
\text { instantáneo }\end{array}$} & \multirow{10}{*}{ Bajo } & Cualidades de los alimentos & Energía & & Instantáneo \\
\hline & & Panadería y pastelería & Galleta & & \\
\hline & & Comida & Desayuno & Desayunar & \\
\hline & & Estados de ánimo & & Disfrutar & \\
\hline & & Ejercicio & $\begin{array}{c}\text { Atleta } \\
\text { Deporte }\end{array}$ & & \\
\hline & & Ropa y calzado & & Calzar & \\
\hline & & Objeto & Masterchef & & \\
\hline & & Edificio & Casa & & \\
\hline & & Persona & Hijos & & \\
\hline & & Ocio & $\begin{array}{c}\text { Cine } \\
\text { Imaginación } \\
\text { Amigos } \\
\text { Aventuras }\end{array}$ & $\begin{array}{l}\text { Jugar } \\
\text { Volar }\end{array}$ & \\
\hline Caldo & Medio & Cualidades de los alimentos & & & $\begin{array}{c}\text { Casero } \\
\text { Natural } \\
\end{array}$ \\
\hline \multirow{4}{*}{ Caramelos } & \multirow{4}{*}{ Bajo } & Cualidades de los alimentos & & Refrescar & \\
\hline & & Chocolates y dulces & Caramelo & & \\
\hline & & Ingerir & & Probar & \\
\hline & & Estados de ánimo & & Sorprender & \\
\hline \multirow{5}{*}{ Cereales } & \multirow{5}{*}{ Bajo } & Cualidades de los alimentos & Sabor & & Delicioso \\
\hline & & Chocolate y dulces & Chocolate & & \\
\hline & & Comida & & $\begin{array}{l}\text { Desayunar } \\
\text { Chocolatear }\end{array}$ & \\
\hline & & Estados de ánimo & $\begin{array}{r}\text { Acción } \\
\text { Placer }\end{array}$ & Flipar & Salvaje \\
\hline & & Ocio & $\begin{array}{c}\text { Plan } \\
\text { Diversión }\end{array}$ & & \\
\hline \multirow{7}{*}{ Chocolate } & \multirow{7}{*}{ Bajo } & Cualidades de los alimentos & Azúcar & & $\begin{array}{c}\text { Delicioso } \\
\text { Bueno } \\
\text { Fácil }\end{array}$ \\
\hline & & Chocolate y dulces & $\begin{array}{c}\text { Cacao } \\
\text { Chocolate }\end{array}$ & & \\
\hline & & Frutos secos & Avellanas & & \\
\hline & & Lácteos & Leche & & \\
\hline & & Comida & $\begin{array}{l}\text { Bocadillo } \\
\text { Desayuno }\end{array}$ & & \\
\hline & & Estados de ánimo & Entusiasmo & & \\
\hline & & Ocio & $\begin{array}{l}\text { Amigos } \\
\text { Fiesta }\end{array}$ & & Divertido \\
\hline
\end{tabular}




\begin{tabular}{|c|c|c|c|c|c|}
\hline $\begin{array}{c}\text { Categorías } \\
\text { de alimentos }\end{array}$ & $\begin{array}{c}\text { Valor } \\
\text { nutricional }\end{array}$ & Campos semánticos & $\begin{array}{c}\text { Unidad léxica: } \\
\text { sustantivo }\end{array}$ & $\begin{array}{c}\text { Unidad léxica: } \\
\text { verbo }\end{array}$ & $\begin{array}{c}\text { Unidad léxica: } \\
\text { adjetivo }\end{array}$ \\
\hline Fruta & Alto & Cualidades de los alimentos & Sabor & & Nuestro \\
\hline \multirow{11}{*}{ Galletas } & \multirow{11}{*}{ Bajo } & Cualidades de los alimentos & $\begin{array}{c}\text { Hierro } \\
\text { Vitaminas } \\
\text { Calcio } \\
\text { Sabor }\end{array}$ & & $\begin{array}{l}\text { Bueno } \\
\text { Dulce } \\
\text { Crujiente } \\
\text { Nuevo } \\
\text { Delicioso } \\
\text { Minis } \\
\text { Más }\end{array}$ \\
\hline & & Cereales & Cereales & & \\
\hline & & Chocolate y dulces & $\begin{array}{l}\text { Chocolate } \\
\text { Dinosaurio }\end{array}$ & & \\
\hline & & Panadería y pastelería & Galletas & & \\
\hline & & Bollería & Huevo & & \\
\hline & & Comida & Desayuno & Desayunar & \\
\hline & & Ingerir & Cucharadas & & \\
\hline & & Partes del cuerpo & Corazón & & \\
\hline & & Acción & $\begin{array}{c}\text { Aprender } \\
\text { Abrir } \\
\text { Mojar }\end{array}$ & & \\
\hline & & Ocio & $\begin{array}{c}\text { Magia } \\
\text { Imaginación } \\
\text { Dibujo } \\
\text { Concurso } \\
\text { Historias } \\
\text { Diversión } \\
\text { Amigos }\end{array}$ & $\begin{array}{l}\text { Cantar } \\
\text { Imaginar }\end{array}$ & \\
\hline & & Asociaciones & $\begin{array}{c}\text { Asociación } \\
\text { Española de } \\
\text { Pediatría }\end{array}$ & & \\
\hline \multirow{9}{*}{ Helado } & \multirow{9}{*}{ Bajo } & Cualidades de los alimentos & Nutrición & & $\begin{array}{c}\text { Refrescante } \\
\text { Bueno } \\
\text { Delicioso } \\
\text { Fácil }\end{array}$ \\
\hline & & Turrones y heladería & Helado & & \\
\hline & & Ingerir & & Morder & \\
\hline & & Partes del cuerpo & Lengua & & \\
\hline & & Estados de ánimo & & Disfrutar & \\
\hline & & Acción & & Pelar & \\
\hline & & Estaciones del año & Verano & & \\
\hline & & Objetos & Regalos & & \\
\hline & & Ocio & Sorpresas & Poder & \\
\hline
\end{tabular}




\begin{tabular}{|c|c|c|c|c|c|}
\hline $\begin{array}{c}\text { Categorías } \\
\text { de alimentos }\end{array}$ & $\begin{array}{c}\text { Valor } \\
\text { nutricional }\end{array}$ & Campos semánticos & $\begin{array}{c}\text { Unidad léxica: } \\
\text { sustantivo }\end{array}$ & $\begin{array}{c}\text { Unidad léxica: } \\
\text { verbo }\end{array}$ & $\begin{array}{c}\text { Unidad léxica: } \\
\text { adjetivo }\end{array}$ \\
\hline \multirow{11}{*}{ Lácteos } & \multirow{11}{*}{ Medio } & Cualidades de los alimentos & $\begin{array}{c}\text { Defensas } \\
\text { Vitaminas } \\
\text { Ayudita } \\
\text { Sabor } \\
\text { Cremosidad }\end{array}$ & $\begin{array}{l}\text { Repetir } \\
\text { Gustar }\end{array}$ & $\begin{array}{c}\text { Poderoso } \\
\text { Bueno } \\
\text { Sabroso } \\
\text { Cremoso } \\
\text { Irresistible }\end{array}$ \\
\hline & & Comida & Postre & & \\
\hline & & Lácteos & $\begin{array}{c}\text { Queso } \\
\text { Leche } \\
\text { Nata }\end{array}$ & & \\
\hline & & Chocolate y dulces & Chocolate & & \\
\hline & & Estados de ánimo & $\begin{array}{l}\text { Sonrisas } \\
\text { Placer }\end{array}$ & $\begin{array}{l}\text { Disfrutar } \\
\text { Cuidarse } \\
\text { Vencer } \\
\text { Conseguir } \\
\text { Luchar } \\
\text { Sorprender }\end{array}$ & \\
\hline & & Ingerir & & Comer & \\
\hline & & Temperatura & & & Frío \\
\hline & & Fisiología & Digestión & & \\
\hline & & Estaciones del año & Verano & & \\
\hline & & Reto & $\begin{array}{l}\text { Desafío } \\
\text { Misión }\end{array}$ & & \\
\hline & & Ocio & $\begin{array}{c}\text { Equipo } \\
\text { Diversión } \\
\text { Autonomía } \\
\text { Superhéroes } \\
\text { Aventura } \\
\text { Sorpresas }\end{array}$ & $\begin{array}{c}\text { Jugar } \\
\text { Explorar } \\
\text { Descubrir } \\
\text { Coleccionar } \\
\text { Aprender } \\
\text { Viajar } \\
\text { Descubrir }\end{array}$ & Divertido \\
\hline Leche infantil & Medio & $\begin{array}{l}\text { Alimentación } \\
\text { Infantil }\end{array}$ & $\begin{array}{l}\text { Lactancia } \\
\text { Bebé }\end{array}$ & & \\
\hline \multirow{6}{*}{ Pasta } & \multirow{6}{*}{ Medio } & Cualidades de los alimentos & & $\begin{array}{c}\text { Pasar } \\
\text { (no se pasa) }\end{array}$ & \\
\hline & & Comida & Ensalada & & \\
\hline & & Pasta & Fideos & & \\
\hline & & Ingerir & & $\begin{array}{l}\text { Comer } \\
\text { Probar } \\
\text { Saborear }\end{array}$ & \\
\hline & & Valor & & & (no) Caro \\
\hline & & Estados de ánimo & & $\begin{array}{l}\text { Cuidarse } \\
\text { Disfrutar }\end{array}$ & \\
\hline \multirow{2}{*}{ Patatas } & \multirow{2}{*}{ Bajo } & Objetos & $\begin{array}{l}\text { Tatoo } \\
\text { Latas }\end{array}$ & & \\
\hline & & Ocio & Películas & Coleccionar & \\
\hline
\end{tabular}




\begin{tabular}{|c|c|c|c|c|c|}
\hline $\begin{array}{c}\text { Categorías } \\
\text { de alimentos }\end{array}$ & $\begin{array}{c}\text { Valor } \\
\text { nutricional }\end{array}$ & Campos semánticos & $\begin{array}{l}\text { Unidad léxica: } \\
\text { sustantivo }\end{array}$ & $\begin{array}{c}\text { Unidad léxica: } \\
\text { verbo }\end{array}$ & $\begin{array}{c}\text { Unidad léxica: } \\
\text { adjetivo }\end{array}$ \\
\hline \multirow{7}{*}{ Paté } & \multirow{7}{*}{ Bajo } & Cualidades de los alimentos & $\begin{array}{l}\text { Hierro } \\
\text { Sabor }\end{array}$ & $\begin{array}{l}\text { Molón } \\
\text { Hechizar }\end{array}$ & $\begin{array}{c}\text { Rico } \\
\text { Natural }\end{array}$ \\
\hline & & Comida & Bocadillo & & \\
\hline & & & Sándwich & & \\
\hline & & Pescado y marisco & Pescado & & \\
\hline & & Estados de ánimo & & Disfrutar & \\
\hline & & Objetos & $\begin{array}{c}\text { Accesorios } \\
\text { Stickers } \\
\text { Minions } \\
\text { Dedotattoos } \\
\text { Marco de foto }\end{array}$ & & \\
\hline & & Ocio & & Coleccionar & \\
\hline \multirow{6}{*}{ Queso } & \multirow{6}{*}{ Medio } & Cualidades de los alimentos & $\begin{array}{l}\text { Sabor } \\
\text { Crema }\end{array}$ & & Bueno \\
\hline & & Estados de ánimo & Campeones & Atreverse & \\
\hline & & Lácteos & Queso & & \\
\hline & & Comida & & Cenar & \\
\hline & & Ejercicio & Deporte & & \\
\hline & & Ocio & $\begin{array}{l}\text { Recreo } \\
\text { Héroe } \\
\text { Amigos }\end{array}$ & & \\
\hline Salchichas & Bajo & Ingerir & & Morder & \\
\hline
\end{tabular}

Fuente: elaboración propia.

Atendiendo a los resultados obtenidos en la tabla 2, observamos que hay una sola categoría de alimentos que cuenta con un valor nutricional alto y que corresponde a fruta, la cual tiene asociado un solo campo semántico, cualidades de los alimentos, en la descripción del producto. También constatamos que hay seis categorías de alimentos que cuentan con un valor nutricional medio y que corresponden a las referencias arroz, caldo, lácteos, leche infantil, pasta y queso. De estas seis categorías, solo una, cal$d o$, hace referencia, exclusivamente, a la cualidad del alimento, y otra, leche infantil, recuerda que es un producto de alimentación infantil. En las cuatro categorías restantes, observamos que los campos semánticos que tienen en común son cualidades de los alimentos (4 categorías), estados de ánimo (4 categorías) y comida( 3 categorías). Además, la categoría lácteos tiene asociados los campos semánticos chocolate y dulces, ingerir, temperatura, fisiología, estaciones del año y reto, y en la categoría de queso se alude a ejercicio. Asimismo, constatamos que los anuncios relacionados con 
la categoría de queso tienden a poner en valor las cualidades del producto (sabor, crema, bueno) y la capacidad de relacionarse con el entorno (recreo, héroe, amigos). Observamos, en cambio, que los anuncios pertenecientes a la categoría de lácteos hacen especial énfasis en los distintos estados de ánimo que se desprenden al consumir el producto (disfrutar, cuidarse, vencer, conseguir, luchar, sonrisas, sorprender, placer) y que establecen una estrecha relación entre el producto y las distintas formas de ocio (equipo, diversión, jugar, explorar, autonomía, descubrir, coleccionar, aprender, viajar, descubrir, divertido, superhéroes, aventura). En dicha categoría, también hemos identificado el uso de creación léxica para presentar los alimentos (Actimelización, actimelízate; Bebedino, Petitdino), lo que en cierto modo es una forma más directa de interactuar con el producto anunciado.

Respecto de las categorías de alimentos que cuentan con un valor nutricional bajo, constatamos que corresponden a diez y son bollería, cacao instantáneo, caramelos, cereales, chocolate, galletas, helado, patatas, paté y salchichas. En estas diez categorías, objetivamos que los campos semánticos que comparten en mayor número son los referentes a ocio( 8 categorías), cualidades de los alimentos ( 8 categorías), estados de ánimo ( 7 categorías), chocolate y dulces, comida e ingerir ( 5 categorías, respectivamente). En ese sentido, podemos aseverar que en estas categorías de alimentos con bajo valor nutricional predomina de forma notable el campo semántico ocio (8 categorías), a diferencia de las categorías que corresponden a un valor nutricional medio, en las que este campo semántico se encuentra presente solo en dos de las seis categorías (lácteos y quesos).

Asimismo, observamos que hay categorías de productos en las que se hace más énfasis en los elementos relacionados con el alimento que en las propias cualidades que este posee. Así, en la categoría cacao instantáneo, se hace referencia solo a las cualidades del producto a través de dos unidades léxicas (instantáneo, energía) y, en cambio, hay una amplia descripción de aspectos extrínsecos al producto como puede ser el momento de consumirlo (desayuno), su aplicación en el ejercicio físico (atleta, deporte), los objetos de regalo que ofrece (Masterchef), el lugar donde se toma (casa), quién lo consume (hijos) y el ocio asociado (jugar, cine, imaginación, vo- 
lar, amigos, aventuras). En la categoría cereales, también se hace referencia de forma puntual a las cualidades del alimento (delicioso, sabor), lo que contrasta con la amplia referencia a otros aspectos del producto como el tipo de cereales (chocolate), en qué momento del día se consume (desayuno), los estados de ánimo que se desprenden (flipar, acción, placer, salvaje) y el ocio relacionado (plan, diversión). En la categoría galletas, por otra parte, hemos identificado un amplio número de campos semánticos relacionados con las cualidades del alimento (hierro, vitaminas, calcio, bueno, dulce, crujiente, nuevo, delicioso, sabor, minis, más), pero también una completa relación de campos semánticos relacionados con el momento de la ingesta (desayuno), la forma de consumirlas (cucharadas), a qué partes del cuerpo beneficia (corazón), distintas acciones relacionadas con las galletas (aprender, abres, mojas) o el ocio asociado (diversión, magia, imaginación, dibujo, concurso, historias, cantar”, amigos, imagina). En estas tres categorías. también hemos identificado creación léxica relacionada directamente con los productos cacao instantáneo (Rollermania), cereales (ChocoDiversión) y galletas (Dinotauro). De forma habitual, hemos constatado que la creación léxica está muy relacionada con los alimentos que tienen regalos asociados, los cuales solo se pueden conseguir al comprar el tamaño más grande del producto; detalle que siempre se especifica en el audio del anuncio.

A diferencia de las tres categorías de alimentos anteriores, objetivamos que hay una categoría de alimentos que no hace alusión alguna a la cualidad del producto (patatas) y solo lo hace a los campos semánticos relacionados con los objetos (tattoo, latas) que se regalan con la compra del producto y el ocio que tiene asociado (coleccionar, películas). Asimismo, constatamos que en la categoría paté se hace énfasis en los objetos que se regalan comprando el producto (accesorios, stickers, minions, dedottatoos, marco de foto).

Si analizamos directamente las unidades léxicas que pertenecen a un mayor número de categorías de alimentos, observamos cuáles son las ideas clave que se transmiten en los anuncios publicitarios presentes en nuestro estudio. Así pues, para describir el único producto de nivel nutricional alto, 
la fruta, se hace referencia a sabor y nuestro, que son cualidades relacionadas directamente con el producto y el lugar de procedencia. En la misma línea, se utilizan las ideas de casero o natural, defensas, vitaminas, para referirse a alimentos de valor nutricional medio, como el arroz, los lácteos, la pasta y el queso. Igualmente, constatamos que se introducen algunas ideas que se sitúan en el campo semántico de las cualidades de los alimentos, pero que no están tan relacionadas con la composición del producto, como pueden ser bueno, sabor o sabroso, ayudita, poderoso, cremoso o cremosidad, irresistible, repetir o gustar. Asimismo, un buen número de unidades léxicas hacen referencia explícita al tipo de comida en la que se encuentran presentes estos productos (cenar, ensalada, postre) y también a los estados de ánimo que se desprenden al consumir el alimento, como disfrutar, atreverse, cuidarse, placer, vencer, conseguir, luchar, sorprender, o también campeones y sonrisas. En este grupo de alimentos de valor nutricional alto y medio. podemos afirmar que se destacan las propiedades intrínsecas junto con los valores extrínsecos que estos pueden llegar a tener.

Respecto de las ideas clave que se transmiten en los alimentos de valor nutricional bajo, estas varían de forma determinante su enfoque respecto de los dos grupos de alimentos mencionados, puesto que hay un predominio de unidades léxicas relacionadas con el ocio. De esta forma, identificamos una especial repetición de ideas relacionadas con el coleccionismo, la diversión y los amigos. También se hace especial referencia a las sorpresas, la participación en concursos y la imaginación, y a ideas relacionadas con estas, como poder, cantar, jugar, volar, películas, magia, dibujo, historias, fiesta, cine, aventuras o plan. Asimismo, los alimentos de valor nutricional bajo tienen asociados algunos atributos relacionados con la cualidad del producto, como puede ser hierro, natural, vitaminas, calcio, sano, energía, azúcar, pero de forma amplia se hace referencia a ideas relacionadas con las cualidades extrínsecas de estos alimentos (sabor, rico, molón, hechizar, refrescante, bueno, delicioso, fácil, dulce, crujiente, nuevo, minis, instantáneo). Finalmente, también encontramos ideas relacionadas con la experiencia personal que despiertan estos alimentos en el consumidor, muy centradas en la acción de disfrutar, flipar, sorprender, o en sensaciones como el entusiasmo, el placer o la vitalidad. 


\section{Conclusiones}

La mayoría de los anuncios vistos por el público infantil español durante 2015 corresponden a alimentos de bajo valor nutricional. De las cien campañas de alimentos analizadas, solo una corresponde a un producto de alto valor nutritivo, concretamente la de Plátano de Canarias. Por otra parte, diecisiete publicitan alimentos de valor nutricional medio; todas ellas corresponden a leche y a derivados lácteos.

La emisión de estos anuncios no se ajusta únicamente al horario de protección infantil, ya que la franja de consumo televisivo de mayor audiencia por parte ese colectivo queda fuera de ese límite. Más allá de ese dato, observando los resultados, podemos señalar que, a menor valor nutricional de los productos anunciados, mayor es la presencia de unidades léxicas $y$, en general, de palabras que transmiten ideas relacionadas con experiencias positivas. La diversión, la felicidad, la aventura o el éxito son algunos de los conceptos que se derivan del lenguaje utilizado en la publicidad de productos de bajo valor nutricional.

El presente estudio también constata que, a menor valor nutricional de los productos publicitados, mayor es el número de verbos que denotan estados positivos y que, asimismo, a menos calidad nutricional, también es mayor el número de adjetivos calificativos vinculados a experiencias positivas. De esa manera, verbos y adjetivos transmiten emociones positivas y actúan como promesa básica del producto, es decir, como el beneficio que el anunciante ofrece al consumidor. En ese sentido, destacamos como ejemplo el hecho de que, si bien los anuncios de leche destacan las cualidades del producto aludiendo a conceptos como la nutrición o el desarrollo, las campañas de derivados lácteos mezclan estas propiedades con estados de ánimo y acciones asociadas a la felicidad, al éxito y a la diversión, hecho que revierte en el uso de verbos y adjetivos a tal efecto.

Cabe señalar en ese sentido que estas estrategias discursivas vulneran el punto 5 del apartado IV de las normas éticas del Código PAOS, concretamente el que hace referencia a la presentación de los productos. Según este, 
la presentación publicitaria de alimentos o bebidas no deberá inducir a error a los menores de hasta 12 años difundida en medios audiovisuales e impresos 0 a menores de 15 años en internet, sobre los beneficios derivados del uso del producto. Entre ellos podrían señalarse, aunque la lista no sea exhaustiva: la adquisición de fortaleza, estatus, popularidad, crecimiento, habilidad e inteligencia.

Tal como hemos visto, la alusión a aspectos como la fortaleza, la felicidad o el triunfo suelen presentarse como beneficio o promesa básica del producto en la mayoría de los anuncios analizados.

Cabe aseverar que el lenguaje utilizado en la publicidad de productos de bajo valor nutricional analizado revierte negativamente en la estrategia de prevención de la obesidad infantil del Gobierno de España, ya que incita a los menores al consumo de dichos productos, ofreciéndoles emociones y experiencias positivas como beneficio de compra. Por otra parte, tal como ya hemos señalado, los elementos hedónicos presentes en el discurso publicitario de ciertos productos influyen favorablemente en el consumo, ya que, a través de la compra de ese producto, el público busca de manera inconsciente obtener esas sensaciones.

Si bien el Código PAOS regula aspectos vinculados al diseño publicitario de alimentos destinados a menores, lo cierto es que las pautas para esta autorregulación no tienen en cuenta el hecho de que el lenguaje utilizado se basa a menudo en cualidades extrínsecas al producto, ofreciendo la recompensa emocional positiva como valor de consumo. En ocasiones, pues, el lenguaje de la publicidad analizada vulnera la propia normativa en ese sentido, ya que los beneficios atribuidos a la bebida o alimento en cuestión no siempre son inherentes a su uso. Dado que los niños son un colectivo en especial sensible a ciertos estímulos publicitarios, esta recompensa puede resultar un factor altamente influyente en el proceso de decisión de compra.

Existe una enorme desproporción entre el número de anuncios que publicitan productos de bajo y medio valor nutricional, y los que promocionan alimentos de alto valor nutritivo. En este sentido, la normativa también resulta insuficiente por no regular el elevado número de anuncios de 
productos que no contribuyen a la lucha contra la obesidad infantil, frente a la práctica inexistencia de publicidad de alimentos saludables.

La normativa española desde el punto de vista del contenido del mensaje publicitario resulta insuficiente también en cuanto al punto 9 del apartado $\mathrm{V}$ de las normas éticas. En él se señala que

la publicidad de alimentos y bebidas dirigida a menores de hasta 12 años deberá expresarse en un lenguaje comprensible para este público y de forma clara, legible y destacada. Con tal fin, si esa información adicional se ofrece a través de una sobreimpresión, se cuidará el tamaño de las indicaciones, el contraste con el fondo, así como su tiempo de permanencia en pantalla.

Si bien es cierto que la información adicional, o las pautas de vida saludable que aconsejan los propios anunciantes aparece sobreimpresa en el tamaño correcto, no podemos dejar de mencionar dos cuestiones - ambas estrechamente vinculadas - que nos parecen relevantes: la primera de ellas es que los niños no solo ven aquella publicidad de productos dirigidos estrictamente a su edad; la segunda es que los niños reciben el impacto de esos anuncios a edades muy tempranas, edades a las que aún no saben leer. Por tanto, nos parece remarcable poner de manifiesto que hay ciertos aspectos del mensaje publicitario que pasan absolutamente desapercibidos al colectivo infantil menor de 6 años, edad en que se empieza a consolidar el proceso de lectoescritura.

Con el fin de evitar ciertas incongruencias entre la normativa yla aplicación práctica del Código PAOS, tal como pone de manifiesto la presente investigación, resulta necesaria una revisión de aspectos como el uso de emociones y experiencias positivas a partir del lenguaje, la forma en que el discurso publicitario integra las recomendaciones de vida saludable en el propio anuncio, o la proporción de anuncios de alimentos de bajo valor nutricional que ofrecen diariamente las cadenas televisivas, entre otras. Por otra parte, es fundamental una mayor implicación por parte de la industria alimentaria española, así como de agencias y anunciantes, con el objetivo de que la autorregulación de los contenidos publicitarios resulte realmente efectiva en la lucha contra la obesidad infantil. 


\section{Referencias}

Arroyo-Almaraz, I. y García-García, F. (2015). Influencia de las imágenes mentales generadas por la publicidad en la mente del público infantil según la naturaleza del estímulo. Palabra Clave, 18(1), 212238. DOI: $10.5294 /$ pacla.2015.18.1.9

Asociación de Usuarios de la Comunicación. (2016). Menores y televisión: panorámica de la audiencia en 2015. Recuperado de http://www. auc.es/?docauc

Baker, J. L., Olsen, L. W. \& Sørensen, T. I. (2007). Childhood body-mass index and the risk of coronary heart disease in adulthood. New England Journal of Medicine, 357(23), 2329-2337. DOI: 10.1056/ NEJMoa072515

Base de Datos Española de Composición de Alimentos. (2006). Presentación. Recuperado de http://www.bedca.net/

Bibbins-Domingo, K., Coxson, P., Pletcher, M. J., Lightwood, J. \& Goldman, L. (2007). Adolescent overweight and future adult coronary heart disease. New England Journal of Medicine, 357(23), 23712379. DOI: $10.1056 /$ NEJMsa073166

Campos, D., Hernández-Torres, J. J., Agil, A., Comino, M., López, J. C., Macías, V. \& Campoy, C. (2016). Analysis of food advertising to children on Spanish television: probing exposure to television marketing. Archives of Medical Science: AMS, 12(4), 799-807. DOI: 10.5114/aoms.2016.60969

Código de autorregulación de la publicidad de alimentos y bebidas dirigida a menores, prevención de la obesidad y salud (PAOS). Recuperado de http://www.aecosan.msssi.gob.es/AECOSAN/docs/documentos/nutricion/Nuevo_Codigo_PAOS_2012_espanol.pdf 
Crovetto, M., Durán, M., Guzmán, M. y Miranda, C. (2011). Estudio descriptivo de la frecuencia y duración de la publicidad alimentaria emitida en la programación de canales de televisión asociados a ANATEL. Revista Chilena de Nutrición, 38(3), 290-299. DOI: 10.4067/S0717-75182011000300005

De Iulio, S. (2010). The construction of fun in food advertisements targeting children. Young Consumers, 11(2), 96-107. DOI: $10.1108 / 17473611011065782$

Ensenyat, A., Palacios, I., Serra-Paya, N. \& Castro-Viñuales, I. (2016). Objective assessment of physical activity during physical exercise sessions in a multidisciplinary intervention for the treatment of childhood obesity. Apunts: Educació Física i Esports, 125, 35-52. DOI: 10.5672/apunts.2014-0983.cat.(2016/3).125.03

Eunjin (Anna) Kim, S. Ratneshwar \& Esther Thorson (2017) Why Narrative Ads Work: An Integrated Process Explanation, Journal of Advertising, 46:2, 283-296, DOI: 10.1080/00913367.2016.1268984

Fuertes-Olivera, P. A., Velasco-Sacristán, M., Arribas-Baño, A. \& Samaniego-Fernández, E. (2001). Persuasion and advertising English: Metadiscourse in slogans and headlines. Journal of Pragmatics, 33(8), 1291-1307. DOI: 10.1016/S0378-2166(01)80026-6

González Díaz, C. (2013a). Autorregulación en la publicidad de alimentos para niños a través de PAOS: un estudio internacional. Cuadernos. info, 32, 59-66. DOI: $10.7764 /$ cdi.32.491

González Díaz, C. (2013b). La publicidad dirigida a niños en el sector de la alimentación: un estudio atendiendo al tipo de producto. Historia y Comunicación Social, 18, 175-187. DOI: 10.5209/rev_ HICS.2013.v18.44235 
Hyun, S. S., Kim, W. \& Lee, M. J. (2011). The impact of advertising on patrons' emotional responses, perceived value, and behavioral intentions in the chain restaurant industry: The moderating role of advertising-induced arousal. International Journal of Hospitality Management, 30(3), 689-700. DOI: 10.1016/j.ijhm.2010.10.008

Jenkin, G., Madhvani, N., Signal, L. \& Bowers, S. (2014). A systematic review of persuasive marketing techniques to promote fod to children on television. Obesity Reviews, 15(4), 281-293. DOI: 10.1111/ obr.12141

Kelly, B., Halford, J. C., Boyland, E. J., Chapman, K., Bautista-Castaño, I., Berg, C., ... \& Grammatikaki, E. (2010). Television food advertising to children: a global perspective. American Journal of Public Health, 100(9), 1730-1736. DOI: 10.2105/AJPH.2009.179267

Kemp, E., Bui, M. \& Grier, S. (2013). When food is more than nutrition: Understanding emotional eating and overconsumption. Journal of Consumer Behaviour, 12(3), 204-213. DOI: 10.1002/cb.1413

Kim, E., Ratneshwar, S. \& Thorson, E. (2017). Why narrative ads work: An integrated process explanation. Journal of Advertising, 46(2), 283296. DOI: $10.1080 / 00913367.2016 .1268984$

Lee, B., Kim, H., Lee, S. K., Yoon, J. \& Chung, S. J. (2014). Effects of exposure to television advertising for energy-dense/nutrient-poor food on children's food intake and obesity in South Korea. Appetite, 81, 305-311. DOI: 10.1016/j.appet.2014.06.103

Martín Llaguno, M. M., Fernández-Poyatos, D. y Ortiz-Moncada, R. (2011). Cinco años de Código PAOS en España: un análisis DAFO. Revista de Comunicación y Salud: RCyS, 1(1), 31-41. Recuperado de http://hdl.handle.net/10045/18179 
McGuire, W. J. (2000). Standing on the shoulders of ancients: Consumer research, persuasion, and figurative language. Journal of Consumer Research, 27(1), 109-114. DOI: 10.1086/314312

Menéndez García, R. A. y Franco Díez, F. J. (2009). Publicidad y alimentación: influencia de los anuncios gráficos en las pautas alimentarias de infancia y adolescencia. Nutrición Hospitalaria, 24(3), 318-325. Recuperado de http://scielo.isciii.es/scielo.php?script=sci_artte xt\&pid=S0212-16112009000300009

Oliver, G., Wardle, J. \& Gibson, E. L. (2000). Stress and food choice: A laboratory study. Psychosomatic Medicine, 62(6), 853-865. Recuperado de https://insights.ovid.com/pubmed?pmid=11139006

Ortiz-Pérez, H., Molina-Frechero, N., Martínez-Barbabosa, I. \& CórdovaMoreno, R. (2015). Contenido nutricional de los alimentos promovidos por el Canal 5 de la televisión mexicana dirigidos a la población infantil. Revista Chilena de Nutrición, 42(3), 260-266. DOI: $10.4067 /$ S0717-75182015000300006

Ponce-Blandón, J. A., Pabón-Carrasco, M.yLomas-Campos, M. M. (2017). Análisis de contenido de la publicidad de productos alimenticios dirigidos a la población infantil. Gaceta Sanitaria, 31(3), 180-186. DOI: $10.1016 /$ j.gaceta.2016.12.008

Prell, H., Palmblad, E., Lissner, L. \& Berg, C. M. (2011). Health discourse in Swedish television food advertising during children's peak viewing times. Appetite, 56(3), 607-616. DOI: 10.1016/j.appet.2011.01.033

Royo-Bordonada, M. Á., León-Flández, K., Damián, J., Bosqued-Estefanía, M. J., Moya-Geromini, M. Á. \& López-Jurado, L. (2016). The extent and nature of food advertising to children on Spanish television in 2012 using an international food-based coding system and the UK nutrient profiling model. Public Health, 137, 88-94. DOI: 10.1016/j.puhe.2016.03.001 
Sevillano, M. L.y Sotomayor, A. (2012). Publicidad y consumo de alimentos en estudiantes de Huánuco (Perú). Comunicar, 20(39), 177184. DOI: 10.3916/C39-2012-03-08

Teodorescu, A. (2015). Linguistic patterns in advertising messages:

Knowledge horizons. Economics, 7(3), 115-118. Recuperado de https://EconPapers.repec.org/RePEc:khe:journl:v:7:y:2015:i: 3:p:115-118 